\title{
The OMC-1 molecular hydrogen outflow as a fragmented stellar wind bubble
}

\author{
Mark J. McCaughrean \& Mordecai-Mark Mac Low \\ Max-Planck-Institut für Astronomie, \\ Königstuhl 17, 69117 Heidelberg, Germany \\ Electronic mail: mjm \& mordecai@mpia-hd.mpg.de
}

Received —

In Press, Astronomical Journal, January 1997 


\begin{abstract}
We present new images of the OMC-1 molecular hydrogen outflow, made using long-slit spectroscopy in order to accurately subtract the underlying continuum emission. These images reveal an extremely clumpy, quasi-spherical inner shell that breaks up at larger radii into bow-shocks and trailing wakes in the north-west, as originally described by Allen \& Burton (1993); a fainter counter-finger to the south-east is newly discovered in the present data. While the outflow appears to be broadly bipolar, this is probably due to an interaction between an initially spherical wind from the source and a large-scale density enhancement surrounding it, rather than direct collimation imposed close to the source. The clumpy appearance of the inner shell confirms the prediction of the recent model of Stone, Xu, \& Mundy (1995), in which a spherical and time-varying wind fragments a swept-up shell, producing high-velocity shrapnel, which in turn drives bow-shocks into the surrounding gas, resulting in the observed "fingers". As an alternative to the single varying source proposed by Stone etal., we speculate that several young sources in the BN-KL cluster may have been responsible for first sweeping up the shell and then fragmenting it.
\end{abstract}




\section{Introduction}

Mass outflow is known to be a common and perhaps inevitable part of star formation, a process more axiomatically associated with mass infall. Observations of young low-mass stars at optical, near-infrared, and millimeter wavelengths often reveal highly collimated bipolar jets and molecular outflows ( $c f$. Edwards, Ray, \& Mundt 1993), generally believed to be driven by magnetocentrifugal forces arising through an interaction between the magnetic field and wind associated with a rotating circumstellar disk (e.g., Königl \& Ruden 1993). The situation for high-mass stars is less clear: although winds are known to be a continuing force throughout the evolution of a massive star ( $c f$. Langer et al. 1994), their role is not well understood at the earliest phases, at least in part because massive stars are intrinsically rather rare and generally quite far from the Sun, making it harder to study them in detail. Nevertheless, bipolar outflows have been found from a few young massive stars including Cepheus A (Bally \& Lane 1991), S 106 (Staude \& Elsässer 1993), IRAS 08159-3543 (Neckel \& Staude 1995), G25.65+1.05 and G240.31+0.07 (Shepherd \& Churchwell 1996). Compared to low-mass systems, their outflows tend to be more chaotic, spreading over a wider angle, and often appearing to have multiple jets. As yet however, the driving mechanisms for the outflows from young massive stars remain unclear.

The nearest site of ongoing massive star formation is in the BN-KL complex embedded in the OMC-1 molecular cloud behind the Orion Nebula (Genzel \& Stutzki 1989). IRc2 has long been thought to be the dominant source of luminosity in the complex, in part because it is the brightest compact source of mid- to far-infrared luminosity, and also because of its association with typical high-mass star formation tracers such as water masers (Wynn-Williams et al. 1984). However, recent high spatial resolution infrared and radio observations have shown the situation to be considerably more complicated (Gezari 1992; Dougados etal. 1993; Menten \& Reid 1995). When viewed at sub-arcsec resolution, 
the nominal point source IRc2 is seen to break up into a small cluster of sources, none of which are directly associated with radio emission from ultracompact H II regions expected of young, massive stars. However, such an ultracompact H II region is found to lie directly south of the infrared sources, suggesting that perhaps the latter are all simply "holes in the clouds" illuminated by the true dominant luminosity source (Menten \& Reid 1995), and that luminosity estimates based on their infrared emission are unreliable. Furthermore, the nearby source "n" (Lonsdale et al. 1982) shows both strong IR emission and radio emission from an ultracompact H II region with a hint of bipolar structure that lies within the error bars for the center of emission for the water masers, unlike IRc2 (Menten \& Reid 1995). Therefore, it seems apparent that the core of the BN-KL complex holds a small cluster of very young self-luminous sources, also including the BN and IRc9 sources (Beck 1984). What remains uncertain is which source, if any, dominates the total luminosity of the complex.

What is certain, however, is that the BN-KL complex is associated with a spectacular outflow. Embedded in OMC-1 and obscured by $A_{V} \sim 20^{m}-50^{m}$, the outflow must be observed at infrared wavelengths or longer: the shocked molecular gas associated with the outflow is best seen in the near-infrared emission lines of $\mathrm{H}_{2}$, notably the $\mathrm{v}=1-0 \mathrm{~S}(1)$ line at $2.122 \mu \mathrm{m}$ (Beckwith et al. 1978; Taylor et al. 1984). The increased spatial resolution achieved by infrared imaging observations over the past decade has revealed a striking swath of bow-shocks and trailed wakes (henceforth referred to collectively as "fingers") originating in the vicinity of IRc2 (Lane 1989; Allen \& Burton 1993; Sugai et al. 1994, 1995; Everett, DePoy, \& Pogge 1995; Schild, Miller, \& Tennyson 1996).

The multiple fingers of shocked $\mathrm{H}_{2}$ cover a wide angle on the sky and therefore pose a problem for most models developed for outflows from low-mass stars, in which only a single jet is thought to be active. It is conceptually possible for a single precessing jet to create 
the multiple fingers, but as the cooling time for excited $\mathrm{H}_{2}$ is short (on the order of one year), the precession speed required to produce multiple fingers would be extraordinary; furthermore, curved jets would be expected (Schild et al. 1996). With these problems in mind, Allen \& Burton (1993) revived a long dormant model for Herbig-Haro objects, in which "bullets" of material are ejected from a central source, forming bow-shocks and shocked wakes behind them as they plow through ambient molecular gas. Allen \& Burton suggested that a single explosive event in the BN-KL complex within the past few thousand years might have resulted in a veritable barrage of "artillery shells" (Burton \& Allen 1994), creating the swath of fingers seen today. However, the bullet model for $\mathrm{H}-\mathrm{H}$ objects, bow-shocks, and wakes had fallen out of favor because of the difficulty associated with ejecting isolated clumps of molecular gas from a source in an explosive event and having them remain coherent over long paths through the surrounding molecular cloud. In the case of OMC-1, there is also the problem that multiple bullets must be ejected almost simultaneously over a wide fan.

Stone, Xu, \& Mundy (1995) recently proposed a new model for creating the bullets and swath of fingers seen in OMC-1. Their model starts with a central, massive, young stellar object driving a strong spherical wind, sweeping up ambient gas into a thin, dense shell. As long as such a shell continues to decelerate, it will remain stable to Rayleigh-Taylor instabilities. However, Stone et al. propose that the wind velocity may vary with time, and that if the velocity were to increase sharply, the shell would be accelerated into the surrounding dense medium, become subject to Rayleigh-Taylor instabilities, break into fragments, and form exactly the swath of bow-shocks and wakes that is observed. Thus, their model achieves three important things: first, fragments are created; second, a single event at the source — the increase in wind speed — naturally leads to multiple fragments over a wide area; and third, these fragments are created at some finite radius from the source, thus allowing them to be observed quite some way from it before being destroyed. 
Moreover, this model predicts that behind the fingers, the inner part of the shell should break up into a highly clumpy structure, a feature that would not be expected in a standard bullet model, and that had not been observed when Stone et al. made their proposal. In this paper, we present new images of the shocked $\mathrm{H}_{2}$ emission in OMC-1 which confirm that just such a clumpy shell lies at the base of the fingers, therefore adding considerable weight to the proposal of Stone et al.

\section{Observations}

All previous images of the $\mathrm{H}_{2}$ emission in OMC-1 have been made in a direct imaging mode, placing a narrow-band filter in front of a single raster-scanning detector or an imaging array. The problem with this technique is subtraction of bright continuum emission in the same band-pass from the BN-KL complex and foreground Orion Nebula. Observations at a nearby continuum wavelength are required, but by necessity must be taken before or after the $\mathrm{H}_{2}$ line observations. Slight changes in the seeing and sky background emission are inevitable, making accurate removal of point and extended continuum sources difficult.

We have taken another approach to the problem, namely long-slit spectroscopy, in which the line and continuum emission at a given point on the sky are measured strictly simultaneously. We used the MAGIC $256 \times 256$ pixel near-IR camera (Herbst et al. 1993) on the Calar Alto $2.2 \mathrm{~m}$ telescope on 20 November 1994 with a pixel size of 0.6 arcsec, and a slit size of $154 \times 1.2$ arcsec. A direct-ruled ZnSe grism was used to cover the whole $K$ band $(2.0-2.4 \mu \mathrm{m})$ at a resolution of 300 , including several lines of molecular hydrogen, ionized hydrogen and helium, as well as adjacent continuum. A data cube $(\mathrm{RA} \times \operatorname{dec} \times \lambda)$ was built up by stepping the slit over the region, one slit width at a time, with an integration time of 60 seconds per position. Equivalent sky observations were made every 30 minutes several arcmin to the west. The total number of on-source slit positions was 120, therefore covering 
a total area of $\sim 2.5 \times 2.5$ arcmin with an effective pixel size of $1.2 \times 0.6$ arcsec. The region is shown in Figure 1 (Plate A) overlaid on a narrow-band continuum (i.e., no line emission from $\mathrm{H}_{2}$ etc.) image with a similar spatial resolution ( 1.5 arcsec FHWM) for comparison; key objects are labeled for reference.

The data reduction process is illustrated in Figure 2 (Plate B). Figure 2a shows a single raw source frame: the vertical lines are from the extended nebular gas and $\mathrm{OH}$ airglow which dominates the sky background at these wavelengths; the horizontal stripes are from compact continuum sources (stars). Figure $2 \mathrm{~b}$ is a single sky frame, showing just the $\mathrm{OH}$ airglow lines. Figure 2c is the result of subtracting a stack of sky frames from the source frame. In this image, the vertical lines are slightly curved, the horizontal lines slightly tilted, and the wavelength dispersion non-linear, due to various instrumental effects. The curvature and dispersion were determined as a function of slit position and wavelength by fitting bright $\mathrm{OH}$ airglow lines in the sky frames, and the continuum tilt was measured from a number of bright stellar spectra. The data were then rectified to give a linear wavelength dispersion, with extended emission lines running down columns and discrete continuum sources across rows. Each image was then divided by a tungsten-illuminated spectral dome flat. Similar data processing was applied to spectra of a bright spectral standard star, which were then extrapolated to form a full spectral image. Finally, each source image was divided by the standard star image, then multiplied by the appropriate black-body curve in order to remove the atmospheric transmission profile. Figure $2 \mathrm{~d}$ shows the final calibrated and rectified source data for one slit position. Several molecular and ionized nebular emission lines are identified for reference, although only the $\mathrm{v}=1-0 \mathrm{~S}(1) \mathrm{H}_{2}$ line is discussed further in this paper.

The spectral images were stacked to form a cube with axes RA, dec, and wavelength, thus allowing us to extract RA $\times$ dec slices at any given wavelength. Three slices straddling 
the $\mathrm{H}_{2} \mathrm{v}=1-0 \mathrm{~S}(1)$ line at $2.122 \mu \mathrm{m}$ were extracted and co-added, weighted by intensity; an equivalent continuum was made by averaging five slices on either side of $2.122 \mu \mathrm{m}$. These two images were bilinearly interpolated up by a factor of $4 \times 2$, in order to give images with equal pixel size (0.3 arcsec) in RA and declination (Figures 3a and 3b; Plate C). The vertical stripes associated with bright point sources arise due to scattering in the camera, but subtract out well as seen below. The effective spatial resolution in these images is $\sim 1.5$ arcsec FWHM. Finally, the continuum image was subtracted from the $2.122 \mu \mathrm{m}$ image to give a pure $\mathrm{H}_{2} \mathrm{v}=1-0 \mathrm{~S}(1)$ line image (Figure 4, Plate $\left.\mathrm{D}\right)$. The locations of the bright Trapezium OB stars, BN, and IRc2 are marked with white circles. Intensity has been scaled logarithmically to enhance faint details, and residual images of some of the brighter stars are visible due to small interpolation errors.

\section{Discussion}

The bow-shocks and wake fingers described by Allen \& Burton (1993) are seen to the north-west in the new $\mathrm{H}_{2}$ image shown in Figure 4, although they are not completely covered. As noted by Schild et al. (1996), there are also a number of short fingers in the south-east; our new data reveals for the first time however, a direct counterpart to the north-west fingers in the form of a large faint bow-shock and wake extending over 80 arcsec (0.17 pc) above the Trapezium OB stars. Inside the fingers, closer to their origin, the $\mathrm{H}_{2}$ emission is extremely clumpy. This feature was predicted by Draine \& McKee (1993) on the basis of the largely invariant $\mathrm{H}_{2}$ line ratios measured with large aperture spectrometers (Brand et al. 1988, 1989). These line ratios should vary considerably from tip to wake of a bow-shock, and thus for them to appear constant, Draine \& McKee (1993) argued that there must be many such bow-shocks within a spectrometer beam, and that the entire

outflow must contain hundreds of clumps of diameter $\lesssim 1$ arcsec or so. The model of Stone 
etal. (1995) naturally predicts such a clumpy shell inside the elongated fingers as shown in Figure 5. (It must be remembered that the model is only a two-dimensional slice through the outflow, while the observed $\mathrm{H}_{2}$ image in Figure 4 shows the full three-dimensional structure projected on the sky.)

This comparison of model and observations brings into focus the vexing issue of the underlying geometry of the OMC-1 outflow. The Stone et al. model starts with an idealized spherically symmetric wind, while in contrast, observations clearly show the real outflow to be elongated along the north-west/south-east axis. Thus it has typically been described as bipolar (e.g., Sugai etal. 1994, 1995; Erickson etal. 1982; Schulz et al. 1995). However, this apparent incompatibility between the data and model can be reconciled by noting that the OMC-1 outflow is probably not intrinsically bipolar: it is unlikely that direct collimation occurs within a few AU of the source as is thought to be the case in low-mass outflows. The region around the nominal IRc2 includes a dense ridge of quiescent gas and dust with a hot core which runs from north-east to south-west, i.e., perpendicular to the dominant high-velocity (hundreds of $\mathrm{kms}^{-1}$ ) outflow axis (Murata et al. 1992; Genzel \& Stutzki 1989). This ridge is associated with a low-velocity (tens of $\mathrm{km} \mathrm{s}^{-1}$ ) outflow seen in molecular lines including $\mathrm{SO}, \mathrm{SiO}, \mathrm{HCO}^{+}$, and CS (Plambeck et al. 1982; Wright et al. 1983; Vogel etal. 1984; Murata et al. 1991), in which clumps of gas are thought to be in the form of an "expanding doughnut" around IRc2 with a radius on the order of thousands of AU (Plambeck etal. 1982). It is likely that the dense ridge and "doughnut" are some remnant of the original molecular cloud structure, with some suggestion that they may also be related to a putative circumstellar disk or torus around the outflow driving source (Murata etal. 1991). It seems as though the initially spherical shell and outflow interact with this large-scale density enhancement, and are then shaped into a more elliptical form, with perhaps some pinching at the waist (Sugai etal. 1994, 1995). Further out, Sugai etal. (1995) suggest that the fingers arise as "some parts of the outflow penetrate the expanding 
shell probably due to some inhomogeneities in the outflow and/or in the shell": it now seems clear that Rayleigh-Taylor instabilities are responsible for the transition from a shell to the fingers, as modeled by Stone etal. and discussed below in Section 4 .

The degree of shaping or effective collimation in the flow is, at present, uncertain, as it depends on the angle of flow with respect to the line-of-sight. Most observational results suggest that the molecular outflow is tilted out of the plane of the sky, with the north-west "lobe" pointing close to the line-of-sight towards the observer (Schulz et al. 1995). This appears to be confirmed by the detection at optical wavelengths of [OIII] emission associated with the tips of the north-west fingers, which are delineated at IR wavelengths by high excitation [FeII] emission (Axon \& Taylor 1984, Allen \& Burton 1993). To be detected in the optical, these tips must be emerging from the OMC-1 molecular cloud into the foreground Orion Nebula, and indeed, the [OIII] emission is highly blue-shifted (Axon \& Taylor 1984). By contrast, the receding south-east "lobe" may be pushing into denser material, accounting for its shorter and fainter appearance. However, based on their mid-IR $\mathrm{H}_{2}$ line profiles, Parmar et al. (1994) suggest the underlying outflow is very nearly in the plane of the sky, so the orientation remains somewhat ambiguous. However, it seems as though the degree of effective collimation in the outflow must be relatively small regardless, i.e., that the outflow has a wide opening angle (cf. Geballe et al. 1986). A highly collimated flow almost along the line-of-sight would be strongly fore-shortened and show few if any long fingers, in contrast to the wide swath of long $\mathrm{H}_{2}$ fingers actually seen. On the other hand, a flow in the plane of the sky should not show high velocity blue shifted emission unless it was again only loosely collimated. Future measurement of proper motions and radial velocities for the $\mathrm{H}_{2}$ fingers will make it possible to determine the degree of collimation and the geometry of the flow with respect to the plane of the sky. 


\section{Analysis}

Stone et al. (1995) found that the bullets produced in their numerical model decelerated rapidly, and therefore they had to invoke a density gradient in the surrounding medium to explain the observed velocities. However, it appears that this rapid deceleration was a consequence of their adopting the extremely high sound speed of $20 \mathrm{~km} \mathrm{~s}^{-1}$ in their ambient gas in order to maintain numerical resolution in the shell modeled by their computations. Next, we discuss the consequences of setting the sound speed to a more reasonable value.

The characteristic time for stopping a dense cloud or bullet is of order $10 t_{c}$, where the cloud-crushing time $t_{c}$ is the time required for a shock to propagate entirely through the cloud, and is given by

$$
t_{c}=\chi^{1 / 2} r_{b} / v_{b}
$$

where $\chi=\rho_{b} / \rho_{0}$ is the ratio of the bullet density to that of the ambient gas, $r_{b}$ is the radius of the bullet, and $v_{b}$ is its velocity through the background gas (Klein, McKee, \& Colella 1994). For an isothermal shock, the compression ratio $\chi$ is given by the square of the Mach number, so $\chi=v_{b_{0}}{ }^{2} / c_{s}{ }^{2}$, where $v_{b_{0}}$ is the initial velocity of the bullets after fragmentation of the shell and $c_{s}$ is the sound speed in the gas. As a strong lower-limit on $v_{b_{0}}$, we can take the present-day greatest bullet velocity of $v_{b} \sim 400 \mathrm{~km} \mathrm{~s}^{-1}$ from Allen \& Burton (1993), from whom we can also take the mass $m_{b}$ of a typical bullet as $10^{-6} M_{\odot}$. The excitation temperature of the CO in the shell lies between 60-90 K (Snell et al. 1984), corresponding to sound speed $c_{s} \sim 0.5 \mathrm{~km} \mathrm{~s}^{-1}$. Magnetic fields could raise the effective sound speed to as much as several $\mathrm{km} \mathrm{s}^{-1}$, but no more.

We assume that the mass $M$ of the gas responsible for the present day high-velocity $\mathrm{CO}$ emission is a rough indication of the mass that was present in the spherical shell just before it fragmented. Also, we assume a radius $R$ of $\sim 20$ arcsec as an estimate of the

present shell size based on the transition from the inner clumpy shell to fingers in the $\mathrm{H}_{2}$ 
image. From these numbers, we can crudely estimate the local ambient density $\rho_{0}$ when the shell fragmented as $\rho_{0}=3 M / 4 \pi R^{3}$. Taking $M=8.2 M_{\odot}$ (Snell et al. 1984) and a distance to the BN-KL complex of 480 pc (Genzel et al. 1981), we find $\rho_{0} \sim 1.6 \times 10^{-18} \mathrm{~g} \mathrm{~cm}^{-3}$.

Finally, we may rewrite the equation for the cloud-crushing time in terms of the typical bullet velocity and mass, shell density, and sound speed, to find

$$
\begin{aligned}
t_{c} & =(3 / 4 \pi)^{1 / 3} v_{b}^{2 / 3} c_{s}^{-5 / 3} \rho_{0}^{-1 / 3} m_{b}^{1 / 3} \\
& \sim\left(1.4 \times 10^{4} \mathrm{yr}\right) v_{400}^{2 / 3} c_{k m}^{-5 / 3} \rho_{-18}^{-1 / 3} m_{-6}^{1 / 3},
\end{aligned}
$$

where $v_{400}=v_{b} /\left(400 \mathrm{~km} \mathrm{~s}^{-1}\right), c_{k m}$ is the sound speed in $\mathrm{km} \mathrm{s}^{-1}, \rho_{-18}=\rho_{0} /\left(10^{-18} \mathrm{~g} \mathrm{~cm}^{-3}\right)$, and $m_{-6}=m_{b} /\left(10^{-6} M_{\odot}\right)$. As Stone et al. found, sound speeds of $20 \mathrm{~km} \mathrm{~s}^{-1}$ will result in rapid deceleration due to the low density of the resulting bullets. However, realistic effective sound speeds of only a few $\mathrm{km} \mathrm{s}^{-1}$ will result in bullets dense enough to propagate much farther than is presently observed without strong deceleration. Therefore, while we do not rule out a density gradient in the molecular cloud core surrounding the outflow (indeed, one is suggested by the shape of the outflow and by the observation of optical emission from the outermost bullets), we find that no gradient is required to explain the bullets and fingers.

It is worth considering the issue of how the $\mathrm{H}_{2}$ emission actually arises in the fingers. Schild et al. (1996) compute the thickness of the observed layer of warm ( $\mathrm{T} \sim 2000 \mathrm{~K}) \mathrm{H}_{2}$ in the structure they call the "north jet", finding it to be around $0.05 \%$ of the finger cross section. They speculate that this thin layer is formed as ambient $\mathrm{H}_{2}$ is entrained by some unseen jet of material, then heated above its dissociation temperature. However, within the Stone et al. (1995) model, there is no jet, and the natural explanation is that behind the high-velocity fragment, an expanding wake shocks ambient $\mathrm{H}_{2}$, which then rapidly cools below observable temperatures (Hollenbach \& McKee 1989). Schild et al. also note that water masers have been observed coincident with fingers and with large proper motions pointing outwards in the same direction as the fingers. Water masers are most easily 
produced by shocks in dense gas (Elitzur, Hollenbach, \& McKee 1989), so this observation is also naturally explained by the fingers being bounded by shocks, as Stone et al. predict.

Therefore, on balance, we find that with suitable minor modifications, the Stone etal. model does a good job of describing how the OMC-1 $\mathrm{H}_{2}$ outflow is formed, with our new images providing further observational support. There is, however, one ad hoc component in the Stone et al. model, namely a spherical wind from the driving source that is strongly time variable. There is little observational evidence for strong spherical winds from massive YSOs on the large scales required (although several massive YSOs are known to have strong winds much closer in; Hoare et al. 1994). It follows that there is also little evidence for the strong time variability required by Stone et al. to accelerate the shell and reverse the effective gravity, leading to Rayleigh-Taylor instabilities and the resulting bullets and fingers.

Schild etal. (1996) suggest that the need for time variability could be avoided if the fingers were produced by the thin-layer instability described by Dgani, Walder, \& Nussbaumer (1993). However, as pointed out by Vishniac (1994), this instability depends on the existence of a bow-shock-like global geometry, and so cannot explain the presence of instabilities at all points on the inner quasi-spherical bubble. The nonlinear thin shell instability described by Vishniac (1994) is potentially relevant as it can fragment even a decelerating shell if the inner, driving shock is radiative (García-Segura, Mac Low, \& Langer 1995), as would be likely at the densities expected within a molecular cloud. However, no scenario has yet been proposed and simulated that can produce the observed high-velocity bullets and lower-velocity clumpy shell solely with the nonlinear thin shell instability.

Returning to the basic model of Stone etal. (1995), we propose a modification that introduces time-variability in an outflow not through variations in a single source, but through non-synchronous formation and evolution of a cluster of sources. As noted in 
Section 1, recent observations have shown that the BN-KL complex appears to contain a small cluster of very young high-mass stars in the vicinity of the nominal IRc2, plausibly an extremely youthful analog of the nearby Trapezium OB sub-group which illuminates the Orion Nebula. Thus, our suggestion is that perhaps the shell or bubble described by Stone etal. was not created by a single stellar wind, but rather by two or more sources in the BN-KL complex at different stages of evolution.

In this scenario, a relatively weak source or group of sources may have swept up the original shell. The sharp increase in wind strength required to drive the Rayleigh-Taylor instabilities might then arise as a single new source (for example, the deeply embedded source illuminating IRc2) collapses, becomes luminous, and drives a powerful new wind into the bubble. The dynamical timescale required to create the present-day $\mathrm{H}_{2}$ fingers is short, on the order of only $\sim 10^{3} \mathrm{yrs}\left(0.2 \mathrm{pc}\right.$ at $\left.200 \mathrm{~km} \mathrm{~s}^{-1}\right)$. Therefore, since the deeply embedded young stars may be up to $10^{4} \mathrm{yrs}$ old, the necessary "desynchronization" is relatively small, and the cluster could still be considered quasi-coeval.

\section{Conclusions}

Our new continuum-subtracted $\mathrm{H}_{2}$ line image of the OMC-1 outflow confirms the clumpy inner shell structure predicted by the time-variable wind model of Stone et al. (1995), and in addition, shows a new bow-shock and wake system to the south-east. The outflow appears to begin with a quasi-spherical wind as in the model of Stone et al., but is subsequently loosely shaped or collimated by a large-scale density enhancement around the entire cluster of wind sources (Sugai et al. 1994, 1995). While the degree of effective collimation and the flow geometry remain uncertain at this time, high-resolution near-infrared imaging in $\mathrm{H}_{2}$ line using NICMOS on the Hubble Space Telescope and/or ground-based adaptive optics should allow us to measure proper motions of the knots, and 
in combination with radial velocities measured from spectroscopy, enable us to create a three-dimensional picture of the outflow. Furthermore, such high-resolution images should allow us to characterize the clumpy structure of the inner shell on scales a full order of magnitude smaller than the present data. The ultimate goal would be to create a "time history" for the outflow, in order to determine which source or sources were responsible for the initial shell and its subsequent fragmentation. In addition, sensitive imaging at mid-infrared wavelengths (10-20 $\mu \mathrm{m}$ and beyond) at high spatial resolution on the new generation of 8-m class telescopes may help better reveal the geometry and energetics of the cluster of potential driving sources themselves.

We would like to thank Tom Megeath for donating the $2.2 \mathrm{~m}$ time used to obtain the data presented here, Jianjun Xu and Jim Stone for providing Figure 5, and Michael Burton, C. Robert O'Dell, Michael Smith, Alain Lioure, Chris Davis, Antonio Chrysostomou, and Karl Menten for interesting and useful discussions on the nature of the $\mathrm{H}_{2}$ outflow in OMC-1 and its driving source. 


\section{REFERENCES}

Allen, D. A. \& Burton, M. G. 1993, Nature, 363, 54

Axon, D. A. \& Taylor, K. N. R. 1984, MNRAS, 207, 241

Bally, J. \& Lane, A. P. 1991, in Astrophysics with infrared arrays, ASP Conf. Ser. 14, 273

Beck, S. 1984, ApJ, 281, 205

Beckwith, S. V. W., Persson, S. E., Neugebauer, G., \& Becklin, E. E. 1978, ApJ, 223, 464

Brand, P W. J. L., Moorhouse, A., Burton, M. G., Geballe, T. R., Bird, M., \& Wade, R. 1988, ApJ, 334, L103

Brand, P. W. J. L., Toner, M. P., Geballe, T. R., Webster, A. S., Williams, P. M., \& Burton, M. G. 1989, MNRAS, 236, 929

Burton, M. G. \& Allen, D. A. 1994, in proc. "Infrared Astronomy with Arrays: The Next Generation", ed. I. S. McLean, (Kluwer, Dordrecht), p61

Dgani, R., Walder, R., \& Nussbaumer, H. 1993, A\&A, 267, 155

Dougados, C., Lena, P., Ridgway, S. T., Christou, J. C., \& Probst, R. G. 1993, ApJ, 406, 112

Draine, B. T. \& McKee, C. F. 1993, ARA\&A, 31, 373

Edwards, S., Ray, T. P., \& Mundt, R. 1993, in Protostars and Planets III, eds. E. H. Levy \& J. I. Lunine, (Tucson: Univ. of Arizona Press), p567

Erickson, N. R., Goldsmith, P. F., Snell, R. L., Berson, R. L., Huguenin, G. R., Ulich, B. L., Lada, C. J. 1982, ApJ, 261, L103

Everett, M. E., DePoy, D. L., \& Pogge, R. W. 1995, AJ, 110, 1295 
García-Segura, G., Mac Low, M.-M., \& Langer, N. 1996, A\&A, 305, 229

Geballe, T. R., Persson, S. E., Simon, T., Lonsdale, C. J, \& McGregor, P. J. 1986, ApJ, 302, 693

Genzel, R., Reid, M. J., Moran, J. M., \& Downes, D. 1981, ApJ, 244, 884

Genzel, R. \& Stutzki, J. 1989, ARA\&A, 27, 41

Gezari, D. Y. 1992, ApJ, 396, L43

Herbst, T. M., Birk, C., Beckwith, S. V. W., Hippler, S., McCaughrean, M. J., Mannucci, F., \& Wolf, J. 1993, Proc. SPIE 1946, ed. A. M. Fowler, p605

Hollenbach, D., \& McKee, C. F. 1989, ApJ, 342, 306

Hoare, M. G., Drew, J. E., Muxlow, T. B., \& Davis, R. J. 1994, ApJ, 421, L51

Klein, R. I., McKee, C. F., \& Colella, P. 1994, ApJ, 420, 213

Königl, A. \& Ruden, S. P., in Protostars and Planets III, eds. E. H. Levy \& J. I. Lunine, (Tucson: Univ. of Arizona Press), p. 641.

Lane, A. P. 1989, in Low Mass Star Formation and Early Stellar Evolution, ed. B. Reipurth, (ESO, Garching), p. 331.

Langer, N., Hamann, W.-R., Lennon, M., Najarro, F., Pauldrath, A. W. A., \& Puls, J. 1994, A\&A, 290, 819.

Lonsdale, C. J., Becklin, E. E., Lee, T. J., \& Stewart, J. M. 1982, AJ, 87, 1819

Menten, K. M. \& Reid, M. J. 1995, ApJ, 445, 157 
Murata, Y., Kawabe, R., Ishiguro, M., Hasegawa, T., \& Hayashi, M. 1991, in Fragmentation of molecular clouds and star formation, eds. E. Falgarone, F. Boulanger, \& G. Duvert, IAU Symposium 147, (Dordrecht: Kluwer), p357

Murata, Y., Kawabe, R., Ishiguro, M., Morita, K., Hasegawa, T., \& Hayashi, M. 1992, PASJ, 44, 381

Neckel, Th. \& Staude, H. J. 1995, ApJ, 448, 832

Parmar, P. S., Lacy, J. H., \& Achtermann, J. M. 1994, ApJ, 430, 786

Plambeck, R. L., Wright, M. C. H., Welch, W. J., Bieging, J. M., Baud, B., Ho, P. T. P., \& Vogel, S. N. 1982, ApJ, 259, 617

Schild, H., Miller, S., \& Tennyson, J. 1996, A\&A, in press

Schulz, A., Henkel, C., Beckmann, U., Kasemann, C., Schneider, G., Nyman, L. A., Persson, G., Gunnarsson, L. G., \& Delgado, G. 1995, A\&A, 295, 183

Shepherd, D. S. \& Churchwell, E. 1996, ApJ, 457, 267

Snell, R. L., Scoville, N. Z., Sanders, D. B., \& Erickson, N. R. 1984, ApJ, 284, 176

Staude, H. J. \& Elsässer, H. 1993, Astron. Astrophys. Rev., 5, 165

Stone, J. M., Xu, J., \& Mundy, L. G. 1995, Nature, 377, 315

Sugai, H., Usuda, T., Kataza, H., Tanaka, M., Inoue, M., Kawabata, H., Takami, H., Aoki, T., \& Hiromoto, N. 1994, ApJ, 420, 746

Sugai, H., Kawabata, H., Usuda, T., Inoue, M. Y., Kataza, H., \& Tanaka, M. 1995, ApJ, 442, 674 
Taylor, K. N. R., Storey, J. W. V., Sandell, G., Williams, P. M., \& Zealey, W. J. 1984, Nature, 311, 236

Vishniac, E. T. 1994, ApJ, 428, 186

Vogel, S. N., Wright, M. C. H., Plambeck, R. L., \& Welch, W. J 1984, ApJ, 282, 685

Wright, M. C. H., Plambeck, R. L., Vogel, S. N., Ho, P. T. P., \& Welch, W. J 1983, ApJ, 267, L115

Wynn-Williams, C. G., Genzel, R., Becklin, E. E., \& Downes, D. 1984, ApJ, 281, 172 


\section{FIGURE CAPTIONS}

Fig. 1.- Narrow-band $2 \mu \mathrm{m}$ continuum image covering $\sim 3.0 \times 3.5$ arcmin of the Orion Trapezium and BN-KL complex. No bright line emission is included in the filter bandpass. The resolution is 1.5 arcsec FWHM. The region covered by the slit-scan spectroscopy is outlined, and the locations of key point sources are labeled (IRc2 is not detected at $2 \mu \mathrm{m}$ ).

Fig. 2.- Illustration of the slit-scan data reduction process. Fig. 2a shows a single raw spectral image taken in the Orion Nebula. Continuum point sources (stars) run across the wavelength dispersion or x-axis, while extended emission line sources (including the sky background $\mathrm{OH}$ airglow emission) run down the slit or y-axis. The slit length is 154 arcsec, and the wavelength range covered is $2.0-2.4 \mu \mathrm{m}$ at $\mathrm{R}=300$. Fig. $2 \mathrm{~b}$ shows an equivalent raw spectral image of blank sky: only the $\mathrm{OH}$ airglow emission lines are now seen. Fig. 2c shows the simple subtraction of the source and sky images, leaving just the stars across the $\mathrm{x}$-axis and and nebula emission down the y-axis. Fig. $2 \mathrm{~d}$ shows the same data after it has been rectified for curvature, tilt, and non-linear wavelength dispersion; flat-fielded; and corrected for atmospheric absorption. Key nebular emission lines are labeled for reference.

Fig. 3.- Image slices from the $\mathrm{RA} \times \operatorname{dec} \times \lambda$ datacube covering the region outlined in Fig. 1 . Fig. 3a shows the average of the three wavelength slices straddling $2.122 \mu \mathrm{m}$, including the $\mathrm{H}_{2} \mathrm{v}=1-0 \mathrm{~S}(1)$ line and underlying continuum emission. Fig. 3b shows an average of five continuum slices either side of $2.122 \mu \mathrm{m}$. Both images have been interpolated by a factor of $4 \times 2$ to give a uniform pixel size of $0.3 \times 0.3$ arcsec. The extended vertical features are artifacts due to scattering of the brightest continuum sources (e.g., the Trapezium OB stars and the BN object) off the slit.

Fig. 4.- The pure $\mathrm{H}_{2} \mathrm{v}=1-0 \mathrm{~S}(1) 2.122 \mu \mathrm{m}$ line emission image (continuum subtracted). The intensity has been logarithmically stretched to show the full dynamic range. The clumpy 
inner shell surrounding IRc2 is clearly seen, as are the bright fingers of Allen \& Burton (1993) extending to the north-west. A fainter corresponding bow-shock and wake is seen to the south-east, passing above the Trapezium. Locations of important continuum point sources are marked.

Fig. 5.- The fragmentation model of Stone et al. (1995). The image is the logarithm of the density distribution in their two-dimensional model, showing the fragmented shell resulting from a time-variable stellar wind. Low densities are black and high densities are white. The model was computed using an Eulerian hydrocode implementing the piecewise parabolic method. Note that this model is a two-dimensional cut through the shell, and cannot be compared directly to the data shown in Figure 4, which is a two-dimensional projection of the three-dimensional OMC-1 outflow. The model bullets driving the outermost bow-shocks would be dense enough to travel indefinitely if they cooled to realistic temperatures, as discussed in the text. 
This figure "McCaughrean_rev.fig1.jpg" is available in "jpg" format from: http://arxiv.org/ps/astro-ph/9611058v1 
This figure "McCaughrean_rev.fig2.jpg" is available in "jpg" format from: http://arxiv.org/ps/astro-ph/9611058v1 
This figure "McCaughrean_rev.fig3.jpg" is available in "jpg" format from: http://arxiv.org/ps/astro-ph/9611058v1 
This figure "McCaughrean_rev.fig4.jpg" is available in "jpg" format from: http://arxiv.org/ps/astro-ph/9611058v1 
This figure "McCaughrean_rev.fig5.jpg" is available in "jpg" format from: http://arxiv.org/ps/astro-ph/9611058v1 